\title{
Cross Correlation by Neurons of the Medial Superior Olive: a Reexamination
}

\author{
RANJAN BATRA ${ }^{1}$ AND TOM C. T. YIN $^{2}$ \\ ${ }^{1}$ Department of Anatomy, University of Mississippi Medical Center, Jackson, MS 39216-4505, USA \\ ${ }^{2}$ Department of Physiology, University of Wisconsin Medical School, Madison, WI 53706, USA
}

Received: 21 July 2003; Accepted: 03 February 2004; Online publication: 17 June 2004

\section{ABSTRACT}

Initial analysis of interaural temporal disparities (ITDs), a cue for sound localization, occurs in the superior olivary complex. The medial superior olive (MSO) receives excitatory input from the left and right cochlear nuclei. Its neurons are believed to be coincidence detectors, discharging when input arrives simultaneously from the two sides. Many current psychophysical models assume a strict version of coincidence, in which neurons of the MSO cross correlate their left and right inputs. However, there have been few tests of this assumption. Here we examine data derived from two earlier studies of the MSO and compare the responses to the output of a computational model. We find that the MSO is not an ideal cross correlator. Ideal cross correlation implies a strict relationship between the precision of phaselocking of the inputs and the range of ITDs to which a neuron responds. This relationship does not appear to be met. Instead, the modeling implies that a neuron responds over a wider range of ITDs than expected from the inferred precision of phase-locking of the inputs. The responses are more consistent with a scheme in which the neuron can also be activated by the input from one side alone. Such activation degrades the tuning of neurons in the MSO to ITDs.

Keywords: auditory neurophysiology, binaural hearing, interaural time differences, coincidence detection, phaselocking

Correspondence to: Ranjan Batra - Department of Anatomy - University of Mississippi - Medical Center - 2500 North State St. • Jackson MS 39216-4505. Telephone: (601) 984-1652 fax: (601) 9841655; email: rbatra@anatomy.umsmed.edu

\section{INTRODUCTION}

Sensitivity to interaural temporal disparities (ITDs), a major cue for the location of a sound in the azimuthal direction, is generally believed to originate in the superior olivary complex (SOC) (reviewed by Kuwada et al. 1997; Yin 2002). Excitatory inputs from the left and right cochlear nuclei converge on neurons of the medial superior olive (MSO). These neurons are thought to act as coincidence detectors, discharging maximally when the inputs from the two sides arrive simultaneously, in accordance with a model first proposed by Jeffress (1948). A clever feature of this model is a graded system of anatomical delay lines in the projection from the cochlear nuclei to the MSO that compensates for the difference in time required for a sound to reach the two ears. As a result, the ITD at which coincidence occurs differs from neuron to neuron and each neuron discharges maximally at a different ITD. Evidence for such delay lines to the MSO has been found in mammals (Beckius et al. 1999; Smith et al. 1993) and in birds (Carr and Konishi 1990; Young and Rubel 1983). Although there has been almost universal acceptance of the Jeffress model, it has come under increasing criticism in recent years (Brand et al. 2002; McAlpine et al. 2001).

Many current psychophysical models of ITD sensitivity are based on coincidence detection (reviewed by Stern and Trahiotis 1997) and assume a strict version of coincidence in which the strength of the response of the binaural comparators is determined by cross correlation of the temporal discharge pattern of the inputs from the two sides. Few physiological tests of this assumption have been performed, however, because of the difficulty in recording from neurons of the MSO. 
Simple tests of the coincidence mechanism involved comparing the timing of the discharge to monaural tone bursts to the ITD that evoked maximum discharge (Crow et al. 1978; Goldberg and Brown 1969; Joris 1996; Spitzer and Semple 1995; Yin and Chan 1990). The coincidence hypothesis predicts that the ITD that evokes maximum discharge should equal the difference in the delay of the response to stimulation of either ear. Such a relationship was indeed observed in neurons of the MSO. These tests demonstrated that neurons of the MSO detect coincident arrival of signals from the two sides, but they did not demonstrate that these neurons cross correlate the inputs to generate their response.

A more refined test of cross correlation in the MSO involved comparing the responses of a neuron to ITDs of random signals, i.e., noise, with the cross correlation of the responses to the same signals delivered monaurally (Yin and Chan 1990). The cross correlation of the monaural responses resembled qualitatively the binaural response for both correlated and uncorrelated noise stimuli.

Another test which used responses to tones as stimuli was performed by Batra et al. (1997b). They pointed out that just as the ITD that evokes maximal discharge in the Jeffress (1948) model is determined by the delays of the responses to stimulation of either ear, the range of ITDs over which the response is elevated should be determined by the variation in these delays. For cross correlation, the mathematical relationship between the variation in delay and the range of elevated ITDs is described by a convolution principle. Batra et al. (1997b) tested whether neurons in the SOC obeyed this principle by comparing the interval of ITDs over which the response of a neuron was elevated to the jitter in phase-locking to stimulation of one or the other ear. The jitter in phaselocking can be expressed as a synchronization coefficient (SC) (Goldberg and Brown 1969). A SC can also be used to describe the variation in discharge rate of a neuron as a function of the interaural-phase difference, because the variation is periodic. The higher such an interaural-phase SC, the more tightly tuned the response is to a particular ITD. The convolution principle implies that the product of the SCs describing phase-locking to monaural stimuli should equal the SC describing dependence on the interaural-phase difference, or equivalently, the ITD (Mardia 1972). Batra et al. (1997b) found that many neurons of the SOC did obey this convolution principle.

A rationale for the convolution principle can be found by considering the process of convolution (or cross correlation) in the frequency domain. Convolving two functions of time (such as trains of action potentials) is mathematically equivalent to multiplying their Fourier transforms. The SC is the amplitude of the first component of the transform. Thus, the output of a neuron that convolves or cross correlates its inputs should have an interaural-phase SC equal to the product of the SCs of the inputs.

The coincidence mechanism implies a relationship between the inputs and outputs of the MSO. However, none of the physiological tests of the coincidence mechanism has measured the inputs directly, but instead they have inferred the properties of the inputs from the responses of the neurons in the MSO, as pointed out by Irvine et al. (1995). It is generally accepted that although the timing of the inputs can be reasonably inferred, their amplitude cannot because of the significant role of subthreshold events, and also perhaps of inhibitory events, in generating the responses of these neurons. Intracellular recordings from the MSO would be a better measure of the monaural inputs but such data are not available.

In this article we reexamine the issue of whether neurons in the MSO cross correlate their inputs by applying the convolution principle to two sets of data from the MSO in the light of computer and analytic models of the coincidence mechanism. The first data set is that of Yin and Chan (1990), the second that of Batra et al. (1997b). Contrary to the previous findings, the models indicate that the coincidence mechanism is not an exact cross correlation of the inputs but is rather a degraded version of it. The models also point to the difficulty of inferring even the timing of the inputs from the postsynaptic response.

\section{METHODS}

\section{Physiological data}

The two sets of data examined in this article come from independent studies of the superior olivary complex (Yin and Chan 1990; Batra et al. 1997a,b). The first set consists of the responses of 12 neurons in the MSO of barbiturate-anesthetized cats for which the relevant comparisons are available. The second data set comprises 28 peak-type single neurons $(N=11)$ and multiunit clusters $(N=17)$ recorded in the SOC of unanesthetized rabbits. Peak-type neurons are those that show a characteristic delay (Rose et al. 1963; Yin and Kuwada 1983) at or near the maximum of their response. Neurons were judged to have a characteristic delay if the mean interaural phases of their responses varied linearly with frequency. Peak-type neurons were the subset of these neurons with a characteristic phase near 0 cycles. They are presumed to arise in the MSO by the convergence of excitatory inputs from the two sides (Kuwada et al. 1997). The recordings used here are the subset for which responses to matching monaural 
and binaural stimuli were acquired, or for which responses to static delays were tested. In addition to the data from these two studies, we have modeled the response of a neuron studied in the MSO of the dog by Goldberg and Brown (1969). For convenience, we refer to all recordings as "neurons" in what follows.

\section{The model}

Our aim in modeling neurons of the MSO was not to emulate the exact characteristics of individual neurons, but rather to understand the relationship among phase-locking of the inputs, phase-locking of the neuron, and the neuron's tuning to ITDs. For this reason, we employed the simplified model of Colburn et al. (1990), which uses two parameters for the characteristics of the neuron: the threshold and the decay time constant. Our version of the model had two inputs: one from the ipsilateral and the other from the contralateral cochlear nucleus. The model neuron was considered to have a normalized potential, such that its resting potential was zero and an action potential on either input caused a unit increment in the potential. The potential constantly decayed toward rest at a rate determined by the decay time constant $\left(\tau_{\mathrm{d}}\right)$, and the neuron fired an action potential when the potential exceeded the threshold. After an action potential, the membrane potential was reset to zero. The initial membrane potential was zero.

The pattern of phase-locking of each input was modeled in a way similar to that used by Colburn et al. (1990) but was normalized differently for ease of interpretation. For a stimulus $\cos (2 \pi f t)$, the driving function was

$$
d(t)=\frac{D \Delta t}{I_{o}(\kappa)} \exp \{\kappa \cos [2 \pi f(t-\tau)]\}
$$

where $f$ is the frequency of the stimulus; $D, \kappa$, and $\tau$ are parameters, $\Delta t$ is the computational step size (100 $\mu$ s in all the simulations performed); and $I_{0}(\kappa)$ is the modified Bessel function of the first kind and order zero. The parameter $\tau$ is the internal delay. The parameter $D$ determines the input rate, and $\kappa$ determines the strength of phase-locking to the input tone. The parameter $\kappa$ is equivalent to $a_{1}$ in the formulation of Colburn et al. (1990). The present normalization removes a strong dependence of the input rate on $\kappa$ (see Mardia 1972). The parameter $\kappa$ is related to the SC of the driving function, $r$, by

$$
r=I_{1}(\kappa) / I_{0}(\kappa)
$$

where $I_{1}(\kappa)$ is the modified Bessel function of the first kind and of order one (Mardia 1972). For purposes of interpretation, $r$ is of more interest than $\kappa$. For this reason, $\kappa$ was not varied directly in the model. Instead, $r$ was varied and the corresponding value of $\kappa$ obtained from a lookup table that was constructed by iterative calculation of $\kappa$ using Eq. (2) for 47 values of $r$, concentrated in the range $\mathrm{r}=0.9-0.995$. For a spontaneous input, $r$ was set to zero. The input to the model neuron also incorporated a generalized version of the 1-ms refractory periods of Colburn et al. (1990) (see below).

Calculations were performed in a discrete stepwise fashion. The occurrence of an action potential at each input in a particular time step was determined from the current value of the driving function $d(t)$ for that input, from the time since the last action potential at that input, and from the refractory period. If the time since the last action potential was greater than $1 \mathrm{~ms}$, the value of $d(t)$ was taken to be the probability of firing and used with a random number generator to determine if an action potential occurred. If the time since the last action potential was less than or equal to $1 \mathrm{~ms}$, the probability of firing was determined by multiplying $d(t)$ by a parameter $\alpha$, with $0 \leq \alpha \leq 1$ (Han and Colburn 1993). For an absolute refractory period, $\alpha$ was zero, and for the relative refractory period of Colburn et al. (1990), $\alpha$ was 0.3 . The parameter $\alpha$ determines the degree to which an input from only one side activates the postsynaptic cell (Colburn et al. 1990) and thus effectively controls the temporal integration of that input.

All simulations were for 2-s stimuli. Occasionally, multiple repetitions were used to increase the duration of the sample. All calculations were performed using Microsoft Excel, running on either a Macintosh G3 or a PC with a Pentium IV processor.

\section{RESULTS}

Our aim was to examine whether the SCs measured from neurons in the MSO were consistent with the notion that these neurons performed a cross correlation of their inputs in order to generate their sensitivity to ITDs. As stated in the Methods section, two sets of data from the SOC were examined here. The SCs measured in the rabbit have been described previously (Batra et al. 1997b), so we first analyzed the responses of the neurons from the cat to determine if they were consistent with the results obtained in the rabbit.

In the cat, numerous responses to monaurally presented tones were collected for each neuron. Figure 1 illustrates the SCs derived from these responses plotted as a function of frequency for two neurons (Fig. 1A, B, top and middle panels, filled circles). In both neurons, the product of the SCs to 


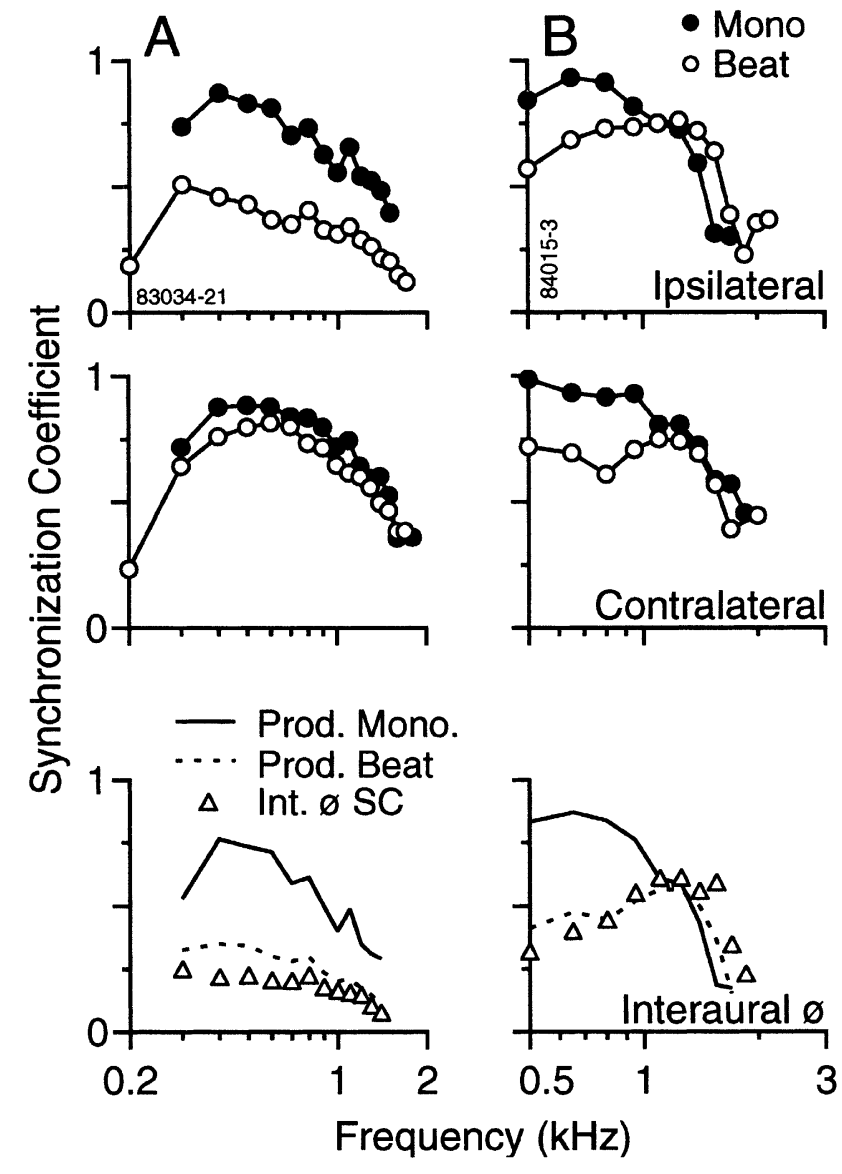

FIG. 1. A,B. Synchrony of two neurons to monaural tones and binaural-beat stimuli as a function of frequency. Both neurons were recorded from the medial superior olive (MSO) of cats by Yin and Chan (1990). Top and middle panels: synchronization coefficients (SCs) to ipsilateral and contralateral tones, respectively, when delivered monaurally (filled circles) or as part of binaural-beat stimuli (unfilled circles). SCs were obtained by Fourier analysis of the responses. Bottom panels: interaural-phase SCs measured in response to binaural-beat stimuli (triangles) and predictions obtained by multiplying ipsilateral and contralateral SCs derived from responses to monaurally presented tones (solid line) and from responses to binaural beat stimuli (dashed line).

monaural ipsilateral and monaural contralateral stimulation (solid line, bottom panels) was poor at predicting the interaural-phase SC (triangles, bottom panels). For the most part, the product of the monaural responses predicted a stronger interaural-phase SC than was actually observed.

Batra et al. (1997b) did not use responses to monaural tones to derive ipsilateral and contralateral SCs. Instead, they extracted these SCs using Fourier analysis of the response to binaural-beat stimuli. A binaural-beat stimulus consists of tones to the two ears that differ slightly in frequency (Kuwada et al. 1979). The difference in frequency results in a cyclically varying change over all possible interaural phases at the difference frequency. Neurons sensitive to ITDs respond over a limited range of interaural phases and therefore synchronize to the difference frequency, reflecting the dependence on the interaural phase difference. The response to a binauralbeat stimulus is also synchronized to the frequencies at the ipsilateral and contralateral ears because most cells in the MSO phase-lock to the low-frequency $(<3$ $\mathrm{kHz}$ ) tones delivered to each ear. The response to this stimulus, therefore, contains components synchronized to the beat frequency and to the frequencies at either ear. Batra et al. (1997b) extracted the SCs for all three components from this response.

For the two neurons of Figure 1, the ipsilateral and contralateral SCs derived from binaural-beat stimuli (unfilled circles, top and middle panels) tended to be less than those derived from monaural tones (filled circles), particularly at lower frequencies. The product of the SCs from the responses to binaural-beat stimuli (dashed line, bottom panels) yielded a better fit to the interaural-phase SCs (open triangles, bottom panels) than those derived from monaural tones (solid line, bottom panels).

The tendency of SCs extracted from responses to binaural-beat stimuli to be less than those extracted from responses to monaural tones was present across all the data in the cat (Fig. 2A, B). At the lowest frequency at which each neuron was studied (unfilled circles, Fig. 2A, B), the difference was statistically significant for both ipsilateral and contralateral SCs (2-tailed $t$-test, $p<0.01)$. At higher frequencies, differences tended to be smaller. In the data from the rabbit (Fig. 2C, D), the differences also tended to be small. The smaller differences in the rabbit are perhaps because such comparisons were typically not available at low frequencies, where differences in the cat seem largest, but only at the best frequency of each neuron.

The reason that ipsilateral and contralateral SCs from binaural-beat stimuli were weaker than the SCs from monaural stimuli did not appear to be because of an overall reduction in SCs to binaural-beat stimuli. In a few cases, responses to matching static delays and binaural-beat stimuli were measured (cat: 9 measurements from 3 neurons; rabbit: 13 measurements from 5 neurons). The interaural-phase SCs derived from binaural-beat stimuli were similar to, or perhaps slightly greater than, the interaural-phase SCs derived from static delays. These observations are in general agreement with the more extensive measurements of Spitzer and Semple (1998).

In the data from the cat, SCs derived from monaural tones were poor at predicting the interauralphase SC (Fig. 3A) and tended to overestimate it. The SCs derived from binaural-beat stimuli (Fig. 3B) were better at predicting the interaural-phase SC but sometimes underestimated it. This was true for nearly all neurons at the lowest frequency tested (unfilled 


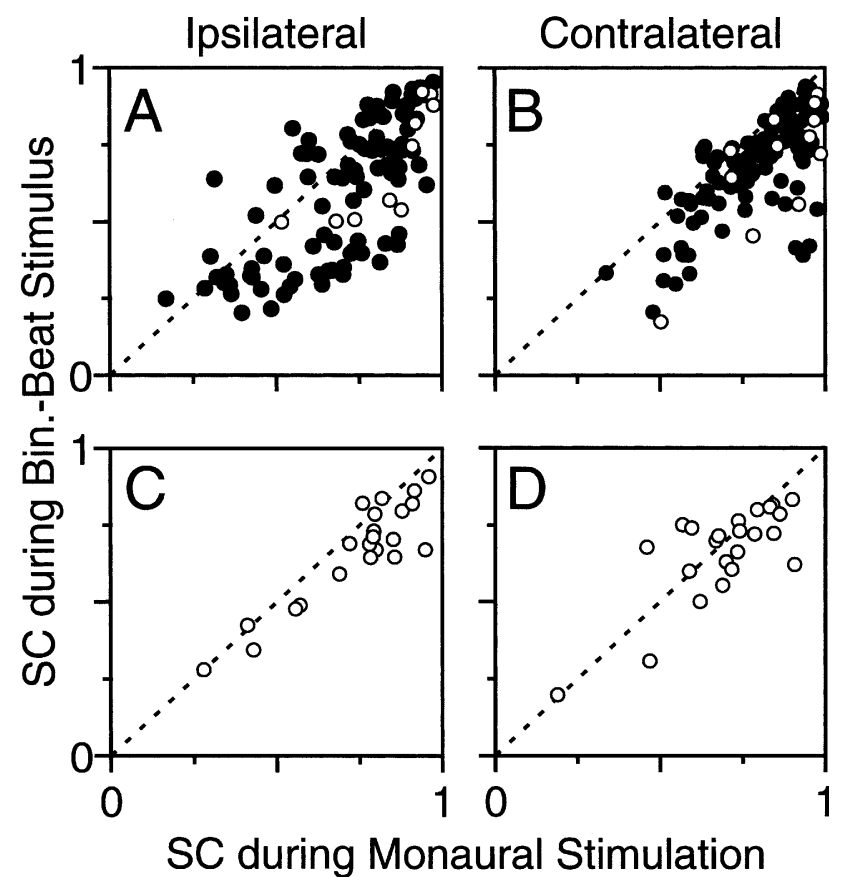

FIG. 2. Comparison of ipsilateral and contralateral SCs derived from responses to binaural-beat stimuli with those derived from responses to monaural stimuli. A,B. Comparisons for neurons in the MSO of the cat. Each point represents responses measured at a particular combination of frequency and intensity. Open circles: response from each neuron at lowest frequency and highest intensity at which comparison was available. C,D. Comparisons for peak-type neurons in the superior olivary complex of the rabbit. Each point represents the response of a different neuron at its best frequency. Dashed lines: equality. A,B: 108 and 127 responses from 10 and 12 neurons, respectively; C,D: 22 and 23 neurons, respectively.

circles, Fig. 3A, B). The improvement in the correspondence between the predicted and measured interaural-phase SCs was reflected in an increase in the correlation coefficient (from 0.42 to 0.72 , using the data at the lowest frequency for each neuron). Indeed, when the single deviant point in Figure 3B (unfilled circle at upper left) was excluded, the correlation was near unity. Monaural SCs measured from binaural-beat stimuli in the rabbit (Fig. 3D) were also better at predicting the interaural-phase SC than those derived from monaural stimuli (Fig. 3C) (correlation coefficients: using monaural SCs from monaural tones, 0.84; using those from binaural-beat stimuli, 0.91).

The observation that the SCs from binaural-beat stimuli were better at predicting the interaural-phase SC than those from monaural stimuli was puzzling. The key question was whether the SCs of the input fibers were better reflected in the response of the neuron during monaural stimulation or during a binaural-beat stimulus. The cross-correlation hypothesis implies that the product of the SCs of the input fibers from the two sides should predict the interaural-phase SC of the MSO neuron. On the one hand, if

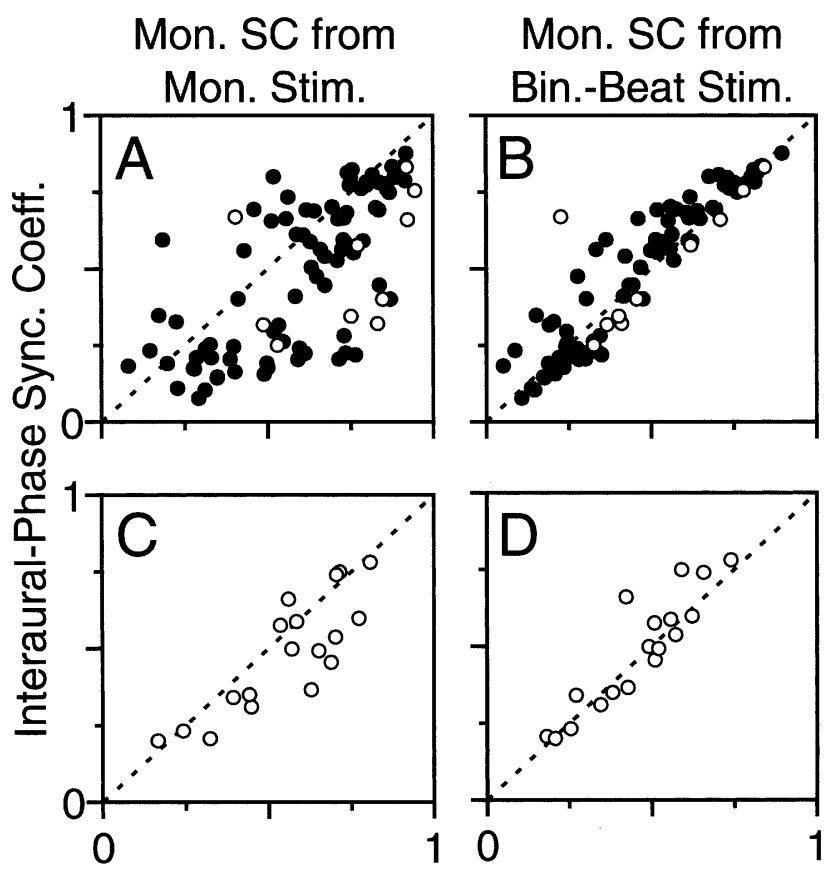

Product of Mon. Sync. Coeff.

FIG. 3. Comparison of the measured interaural-phase SCs with predictions obtained by multiplying ipsilateral and contralateral SCs. Same format as Figure 2. Only responses to matching monaural and binaural-beat stimuli are plotted. Correlation coefficients for full data from cat and for data at lowest frequencies were (A) $0.68,0.42 ;$ (B) $0.85,0.72$. Correlation coefficients for rabbit were (C) 0.84 ; (D) 0.91 . A,B: 97 responses from 10 neurons; C,D: 18 neurons.

SCs from monaural stimuli better reflected the SCs of the inputs, then the data would imply that the neurons were not ideal cross correlators. On the other hand, why did SCs derived from binaural-beat stimuli do so well at predicting interaural-phase SCs? Was it because SCs from binaural-beat stimuli are a more accurate reflection of the SCs of the inputs? To shed light on these questions, we tested a model that had already been shown to be capable of emulating the responses of neurons in the MSO (Colburn et al. 1990).

We began by examining the behavior of the model when it was set to simulate the neuron studied by Goldberg and Brown (1969) (Fig. 4) and modeled by Colburn et al. (1990). The earlier modeling provided two advantages: First, it provided a check on our calculations and, second, we could examine the application of the convolution principle on a modeled neuron using parameters that were chosen for other purposes. We used parameters equivalent to Colburn et al. (1990) (Table 1). However, instead of simulating the static delays they used, we simulated the response to a binaural-beat stimulus, which was never used by Goldberg and Brown (1969).

The model neuron responded to the binaural-beat stimulus with a cyclic variation in the discharge rate. 

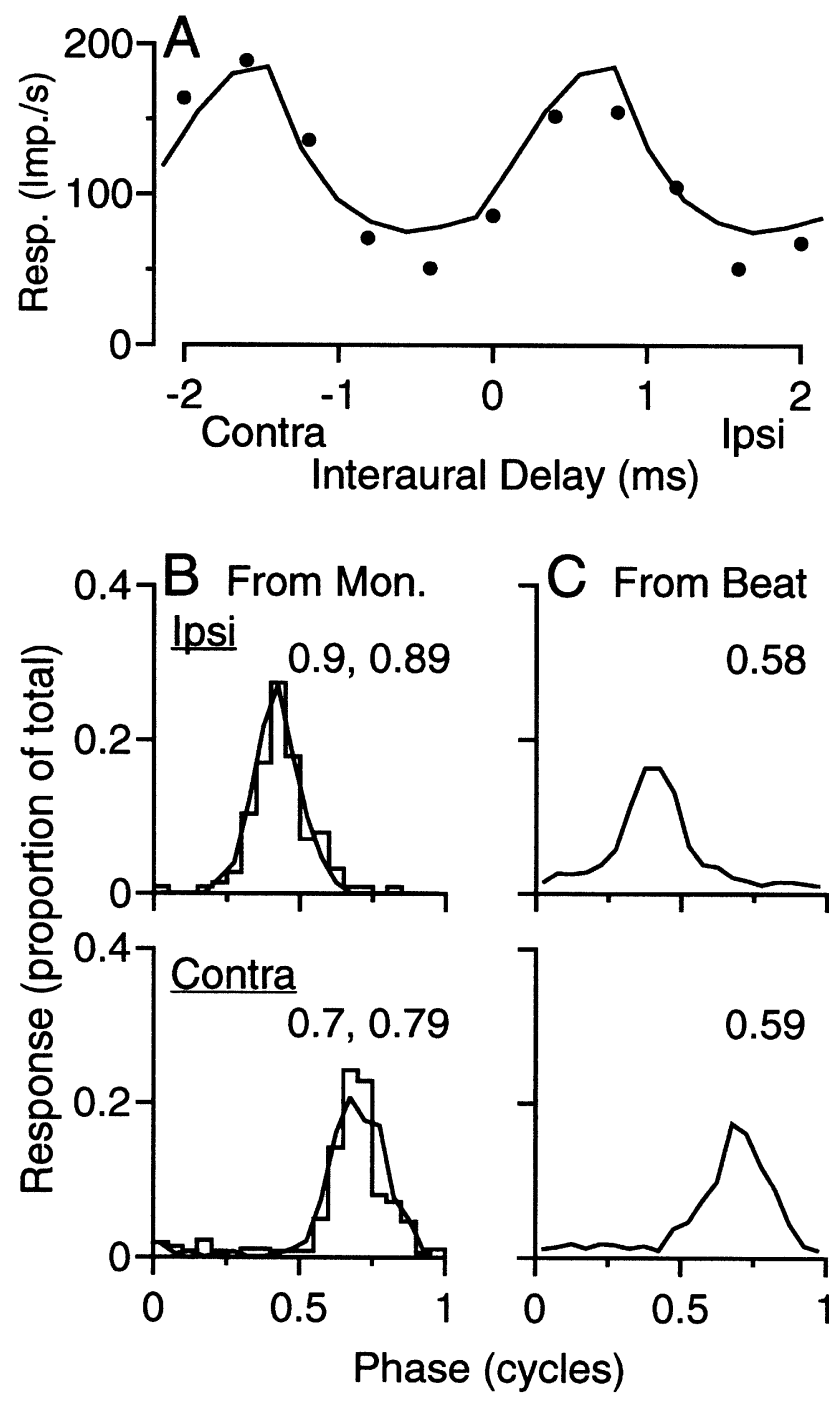

FIG. 4. Modeling of neuron in MSO studied by Goldberg and Brown (1969). A. Comparison of delay curve produced by model (line) with measured response of neuron (circles). B,C. Monaural cycle histograms derived from responses to monaural tones and from binaural-beat stimuli, respectively. Histogram: response of neuron. Smooth lines: response of model. Numbers in left panels: SCs of neuron and model, respectively. Numbers in right panels: SCs of the model. Parameters of model are as given in Table 1 .

When plotted against the equivalent ITD (Fig. 4A, line), the variation in rate matched that observed by Goldberg and Brown (1969) using static ITDs (Fig. 4A, circles). The interaural-phase SCs of the response measured by Goldberg and Brown (1969) and that produced by the model were similar (Fig. 5A, left column, solid and dashed arrows, respectively).

The model also simulated the cycle histograms to the ipsilateral and contralateral tones derived from monaural stimulation that were measured by Goldberg and Brown (1969) (Fig. 4B). The ipsilateral SC from the data and from the model were similar (Fig. 4B, upper panel), but the contralateral SC from the model was slightly higher than that measured (Fig. 4B, lower panel). All the values of the monaural SCs of the binaural cell clustered around the SC of the model input fibers (0.80, cycle histograms not shown).

We next compared the interaural-phase SC of the neuron and the model to predictions of the interaural-phase SC based on the convolution principle (Fig. 5A). In Figure 5A, the solid arrows represent interaural-phase SCs measured from, or based on applying the convolution principle to, neural data, whereas the dashed arrows are interaural-phase SCs that arise from the model. The product of the ipsilateral and contralateral SCs (Fig. 5A, middle column) did not correspond well with the measured interaural-phase SC (0.30), regardless of whether the monaural SCs were derived from the measured responses to monaural tones $(0.63)$, from the response of the model neuron to monaural tones $(0.70)$, or from the input fibers of the model (0.64) (not shown).

Monaural cycle histograms and SCs derived from binaural-beat stimuli are not available in the data of Goldberg and Brown (1969). However, we could obtain them from the model (Fig. 4C). These SCs were weaker than those based on monaural stimulation and those of the input fibers, and their product (0.34) was close to the interaural-phase SC (Fig. 5A, right column).

We also examined the simulation by Colburn et al. (1990) of a second neuron (Fig. 6), which was studied by Yin and Chan (1990). Our parameters are equivalent to theirs, except that the internal delays have been set using the mean phases of the responses to ipsilateral and contralateral tones (Table 2). Also, Colburn et al. (1990) did not simulate the responses of this neuron to monaural tones, so they did not specify a level of spontaneous drive from the unstimulated side which is required to accurately simulate the discharge rate. However, our interest in these responses was chiefly in their temporal aspects, so we selected rates that elicited an adequate number of action potentials for analysis.

As in the previous simulation, the response of the model neuron to a binaural-beat stimulus (Fig. 6A, line), was similar to the response of the neuron to static ITDs (Fig. 6A, circles). It was also similar to the measured response to the binaural-beat stimulus (dashed line) which was recorded by Yin and Chan (1990) but not reported in the original paper. This neuron synchronized strongly to a tone at either ear when it was presented alone (Fig. 6B, histogram) or as part of a binaural-beat stimulus (Fig. 6C, histogram). The model neuron (Fig. 6B, C, lines) mimicked the synchrony to tones, especially for the binaural-beat stimuli (Fig. 6C), but, for responses to monaural stimuli, the SCs of the model were a bit 
TABLE 1

Parameter values for simulation of Goldberg and Brown (1969) Neuron

\begin{tabular}{lcccc}
\hline Stim. & Freq. $(\mathrm{Hz})\left(I_{p} / C_{t}\right)$ & $D\left(s^{-1}\right)\left(I_{p} / C_{t}\right)$ & $r\left(I_{p} / C_{t}\right)$ & $\tau(m s)\left(I_{p} / C_{t}\right)$ \\
\hline Beat & $444 / 445$ & $536 / 536$ & $0.79 / 0.79$ & $0.79 / 0$ \\
Ipsi & 444.5 & $536 / 90$ & $0.900 / 1.575$ \\
Contra & 444.5 & $300 / 536$ & $0.900 /-79$
\end{tabular}

Decay $=200 \mu \mathrm{s}$

Threshold $=1.25$

Relative refractory period $(\alpha=0.3)$
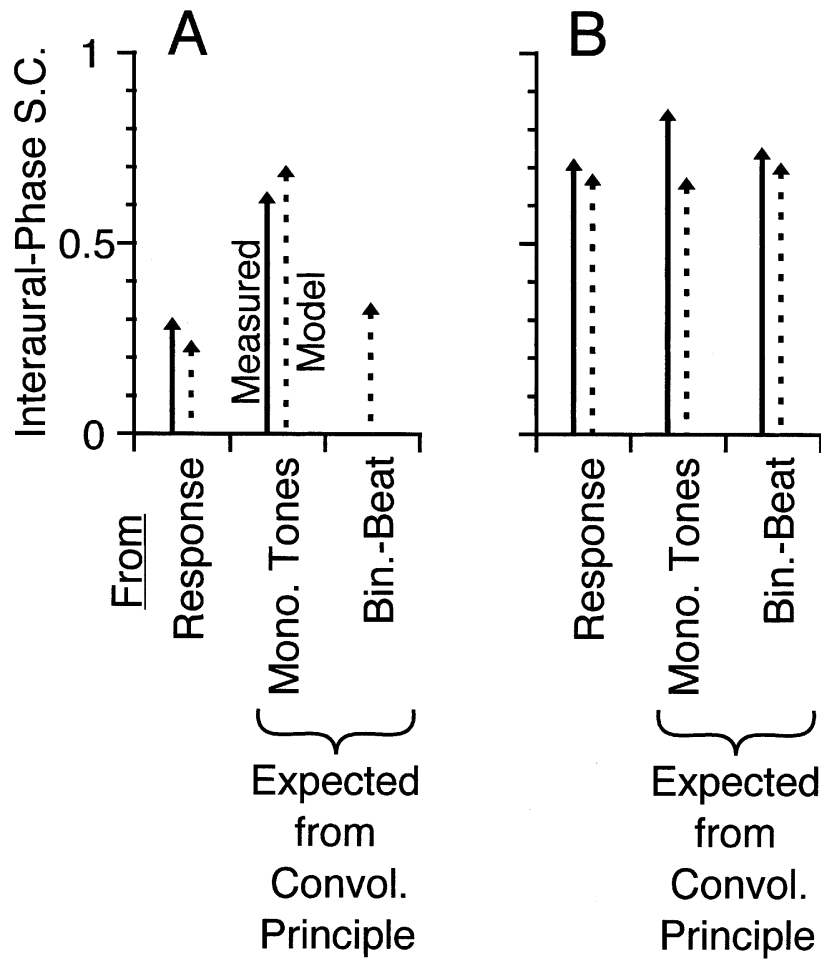

FIG. 5. Modeling of interaural-phase SCs. A. Neuron studied by Goldberg and Brown (1969). B. Neuron studied by Yin and Chan (1990). Solid arrows: interaural-phase SCs measured from, or based on applying the convolution principle to, neural data. Dashed arrows: interaural-phase SCs that arise from the model. Leftmost column in each panel: interaural-phase SC of the response. Interauralphase SC of the neuron was calculated from the response to static delays, that of the model from the response to a binaural-beat stimulus. Center and right columns: expected values of the interaural-phase SC obtained by multiplying the ipsilateral and contralateral SCs derived from responses to monaural tones and from responses to binaural-beat stimuli. Parameters as given in Tables 1 and 2 .

smaller than those of the neuron (Fig. 6B). The SCs derived from both types of responses and from the model were similar to the SCs of the input fibers to the model neuron (0.88) (not shown). The product of the ipsilateral and contralateral SCs derived from the model $(\sim 0.7)$ was close to the measured interaural-phase SC (0.72), as was the product of the
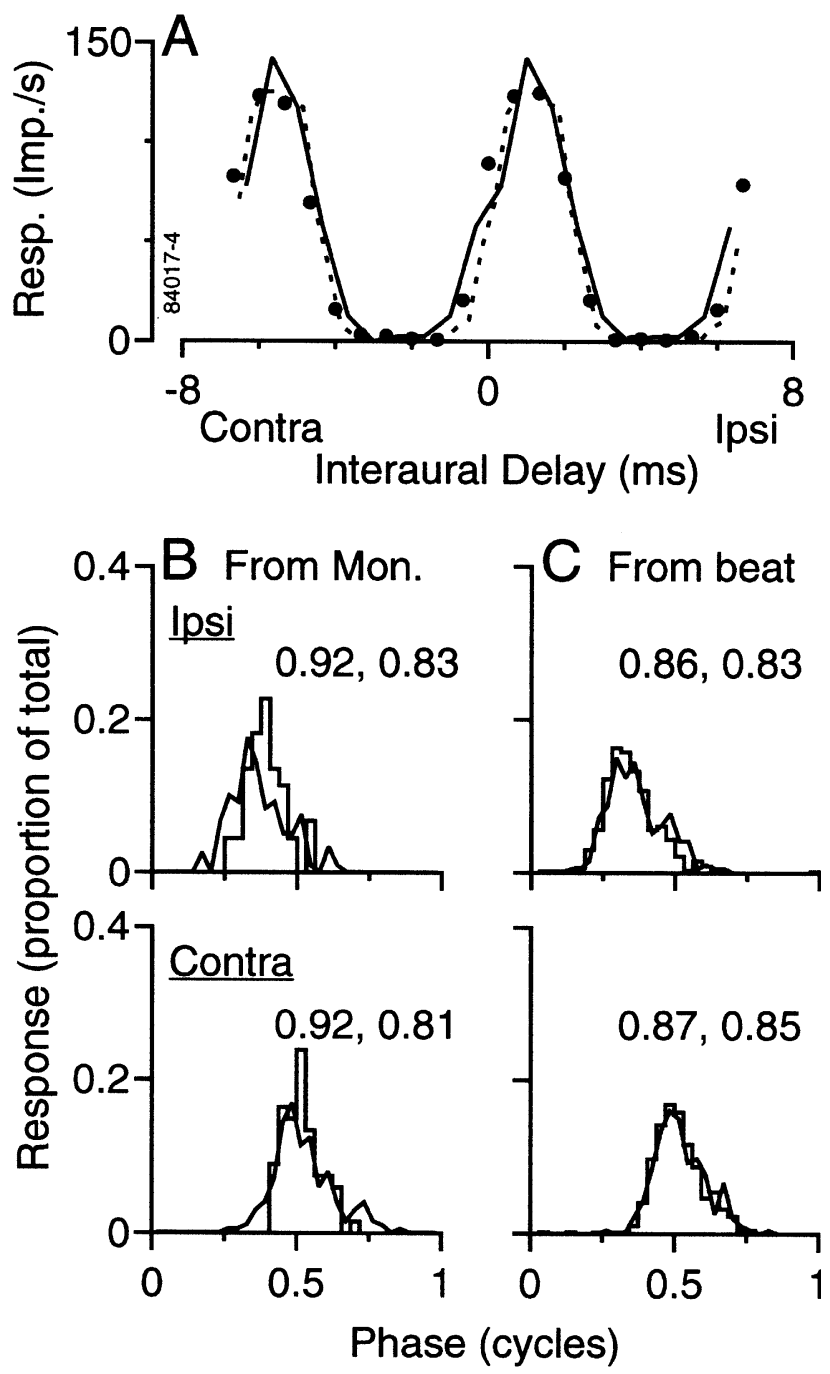

FIG. 6. Modeling of neuron in MSO studied by Yin and Chan (1990). Format similar to that of Figure 4. Dashed line in A: Delay curve derived from response of neuron to binaural-beat stimulus. Parameters of model are as given in Table 2.

measured SCs derived from binaural-beat stimuli (0.75) (Fig. 5B). The product of the SCs derived from responses to monaural tones (0.85) was slightly greater than the measured interaural-phase SC. 
TABLE 2

Parameter values for simulation of Yin and Chan (1990) Neuron

\begin{tabular}{|c|c|c|c|c|}
\hline Stim. & Freq. $(H z)\left(I_{p} / C_{t}\right)$ & $D\left(s^{-1}\right)\left(I_{p} / C_{t}\right)$ & $r\left(I_{p} / C_{t}\right)$ & $\tau(m s)\left(I_{p} / C_{t}\right)$ \\
\hline Beat & $149 / 150$ & $422 / 422$ & $0.91 / 0.91$ & $2.400 / 3.467$ \\
\hline Ipsi & 150 & $422 / 120$ & $0.91 / 0$ & $2.400 /-$ \\
\hline Contra & 150 & $200 / 422$ & 0/0.91 & $-/ 3.467$ \\
\hline
\end{tabular}

Decay $=615 \mu \mathrm{s}$

Threshold $=1.25$

Absolute refractory period $(\alpha=0)$

Simulation of the first neuron (the one studied by Goldberg and Brown 1969) indicated that the model was capable of replicating the observed difference between SCs derived from monaural and binauralbeat stimuli. However, the model behaved differently in the second simulation in that there was no reduction in the monaural SCs during a binaural-beat stimulus (although a small reduction was present in the physiological data). This implies that the parameters controlling the difference in SCs between the two kinds of stimuli differ in the two simulations. Comparison of the parameters in Tables 1 and 2 suggests that $\alpha$, the strength of the refractory period of the inputs, may be a key factor. Colburn et al. (1990) have shown that this parameter controls the degree to which the model neuron will discharge in response to activation of inputs from only one side, i.e., the proportion of so-called "monaural coincidences." In the second simulation, the less-thanperfect fit to the SCs during monaural tones may have been due to an inaccurate value for $\alpha$.

We investigated whether the parameter $\alpha$ could differentially influence monaural SCs derived from responses to monaural and binaural stimuli (Fig. 7A). Starting with the simulation of Figure 6 , the value of $\alpha$ was increased from zero (absolute refractory period) to 0.9 (weak relative refractory period). The ipsilateral SC derived from the response to a monaural tone remained relatively constant (Fig. 7A, unfilled circles), and was similar to the $\mathrm{SC}$ of the input fiber (Fig. 7A, filled circles). In contrast, the ipsilateral SC derived from the response to a binaural-beat stimulus declined (Fig. 7A, triangles). The contralateral SCs behaved similarly (not shown).

Increasing the value of $\alpha$ from zero also caused a decrease in the interaural-phase SC of the model neuron (Fig. 7B, filled circles) and a smaller decrease in the product of the monaural SCs derived from binaural-beat stimuli (Fig. 7B, triangles). In contrast, there was a slight increase in the product of the monaural SCs derived from responses to monaural stimuli (Fig. 7B, open circles). Thus, increasing the value of $\alpha$ from zero resulted in a difference between

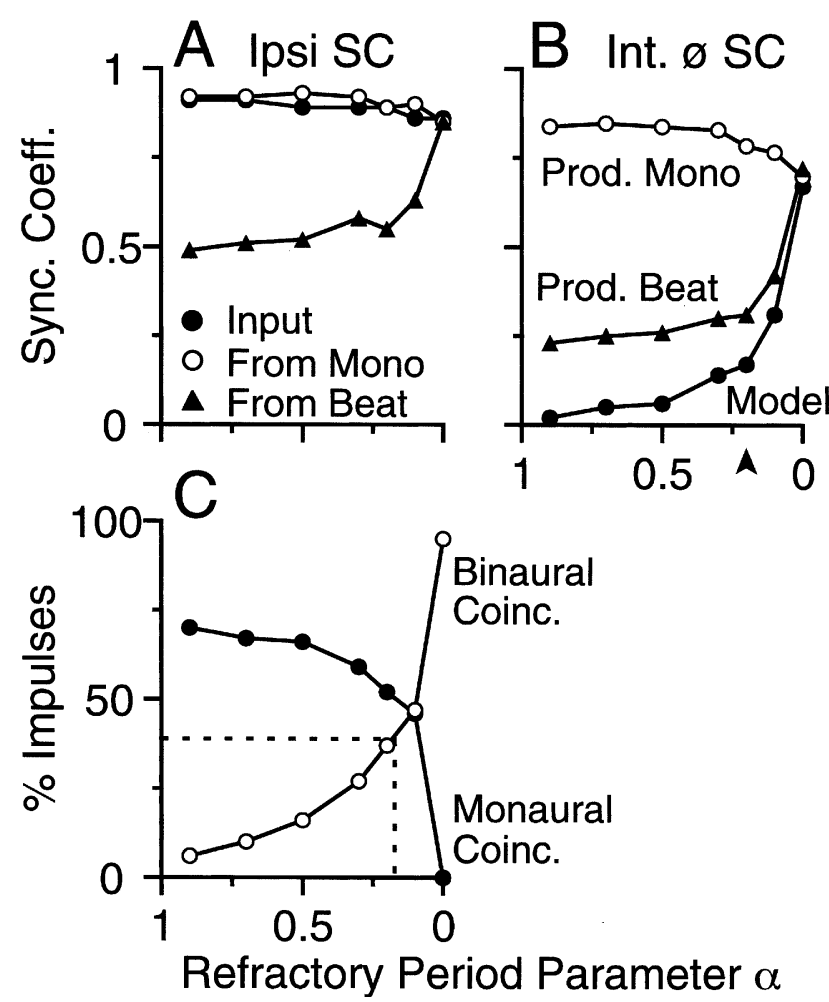

FIG. 7. Effect on the model of changing the refractory period parameter $\alpha$. The parameter $\alpha$ is plotted on a reversed axis because the strength of the refractory period varies inversely with $\alpha$. A. Effect on the ipsilateral SC. Filled circles: SCs of the input fiber; open circles and triangles: SCs derived from responses to monaural and binaural-beat stimulation, respectively. B. Effect on the interauralphase SC. Filled circles: interaural-phase SC of model neuron; open circles and triangles: expected interaural-phase SCs based on monaural SCs derived from responses to monaural and binaural-beat stimulation, respectively. Arrowhead: apparent maximum value of $\alpha$ in neurons studied in cat and rabbit as derived from panel C. C. Effect on source of action potentials in model neuron. Filled circles: monaural coincidences; open circles: binaural coincidences. Dashed lines: lowest apparent proportion of binaural coincidences in cat and rabbit and associated value of $\alpha$. Values of unvaried parameters in simulation are given in Table 2 .

the monaural SCs derived from responses to the two types of stimuli. The SC from the response to monaural stimulation more closely reflected the input but 
was poorly related to the interaural-phase SC except when $\alpha$ was zero. The SC from the response to a binaural-beat stimulus was typically weaker than that of the input but was more closely related to the interaural-phase SC.

In order to determine how an increase in $\alpha$ led to a decline in the interaural-phase SC, we examined the action potentials arriving at each input just before the model neuron fired each of its action potentials. The postsynaptic action potentials were then classified based on whether action potentials were present at only one or at both inputs. If action potentials were present at only one input, the postsynaptic action potential was considered to be a result of "monaural coincidence," whereas if one action potential was present at each input the postsynaptic action potential was considered to be a result of "binaural coincidence." If both inputs were activated and multiple action potentials were present at one or both inputs, the postsynaptic action potential was not classified. Few action potentials ( $\lesssim 5 \%$ ) were elicited by such complex activation when realistic values of parameters were employed. The time interval preceding a postsynaptic action potential that was used for this classification was equal to twice the decay time constant of the neuron.

The decline in the interaural-phase $\mathrm{SC}$ with increasing $\alpha$ was traced to the smaller proportion of action potentials in the binaural neuron that was produced by binaural coincidences (Fig. 7C, unfilled circles). Instead, a larger proportion was a result of monaural coincidences (filled circles), which do not contribute to binaural interaction.

We used a simplified analytic model to examine whether the decline in the proportion of action potentials resulting from binaural coincidences could explain why monaural SCs derived from responses to binaural-beat stimuli were better at predicting the interaural-phase SC (Figs. 8 and 9). As in the model of Colburn et al. (1990), the MSO neuron in this model receives one excitatory input from each side. Figure 8 illustrates the response of the neuron in this model to monaural stimulation. Such stimulation results in activity which is synchronized to the tone arriving at the input on the stimulated side and in spontaneous activity on the other side which is not synchronized to the tone (Fig. 8B). The synchronized activity has an SC denoted by $r_{i}$. The model assumes that the EPSPs are subthreshold so that more than one incoming action potential is required to elicit an action potential from the neuron. Action potentials in the model neuron can be generated in three different ways (Fig. 8C): two or more action potentials arriving at the input on the stimulated side; an action potential arriving at each input; and two or more spontaneous action potentials arriving at the
A

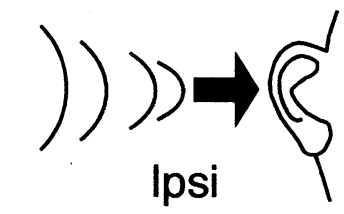

B
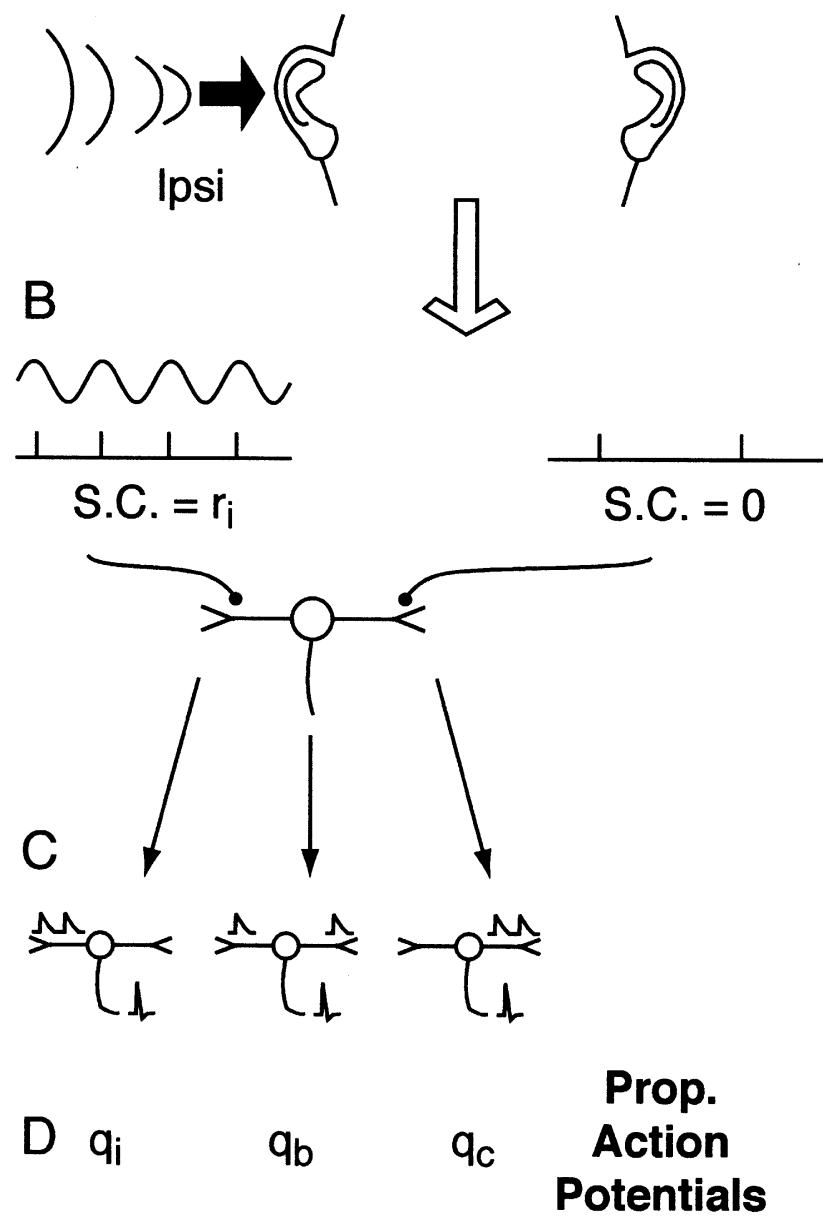

$E s_{i} \quad r_{i} \quad 0 \quad \begin{gathered}\text { Component } \\ \text { SCs }\end{gathered}$

F Aggregate SC

$$
r_{i m}=q_{i} s_{i}+q_{b} r_{i}+q_{c} \times 0
$$

FIG. 8. Analysis of SC in response to monaural stimulation. A. Monaural stimulation. B. Inputs to binaural neuron. Activity of ipsilateral input is synchronized to tone with SC as indicated. Spontaneous activity is present at contralateral input and is not synchronized to the tone. $\mathbf{C}$. Action potentials of the binaural neuron divided into those elicited by ipsilateral monaural coincidences, binaural coincidences, and contralateral monaural coincidences. D. Proportions of action potentials elicited in the three different ways. $\mathbf{E}$. SCs for each group of action potentials. F. The aggregate SC is a linear combination of the SCs of the three components.

unstimulated input (Fig. 8C, left, center, and right panels). Situations in which multiple action potentials arrive along both inputs are assumed to occur infrequently and are ignored. The proportions of the action potentials elicited by each circumstance will, in general, be different (Fig. 8D). 
If the three groups of action potentials are considered separately, then an SC can be associated with each (Fig. 8E). The SC for action potentials arriving at the unstimulated input is zero. The $\mathrm{SC}$ of the action potentials generated by binaural activation will be close to $r_{i}$. This is because, in effect, the spontaneous activity on the unstimulated side randomly samples the activity on the stimulated side at different phases. The output SC is reduced somewhat relative to $r_{i}$ because of the decay time constant and the threshold. The amount of reduction depends on the relationship between the maximum interval between incoming action potentials that can raise the membrane potential of the neuron above threshold and the stimulus period. For the Colburn et al. (1990) modeling of both the Goldberg and Brown (1969) neuron and the Yin and Chan (1990) neuron, this "coincidence interval" is $\sim 0.12$ cycle. Based on the SCs of the incoming action potentials in the modeling of the Goldberg and Brown (1969) neuron and the Yin and Chan (1990) neuron (0.80 and 0.88, respectively, not illustrated), the inputs are distributed over $\sim 0.21$ cycle and $\sim 0.16$ cycle, respectively ( \pm 1 SD, see Mardia 1972). Thus, for these two neurons the coincidence interval is less than the interval over which incoming action potentials are distributed and little reduction will occur.

Lastly, the SC of the action potentials elicited by monaural activation from the stimulated side $\left(s_{i}\right)$ is somewhat greater than $r_{i}$, the SC of the input, because generation of the action potential in the neuron requires coincident arrival of two or more action potentials at that input, and this is most likely when all the action potentials occur near the preferred phase of the incoming synchronized activity. Again, any reduction in the SC due to the decay time constant is slight.

The aggregate $\mathrm{SC}$ of this model neuron depends linearly on the SCs of the three components (Fig. 8F), because all components have the same, or nearly the same, mean phase (see Appendix). The relative contributions of the three components will depend on the relative firing rates at the two inputs

FIG. 9. Analysis of the SCs in response to a binaural-beat stimulus. A. Binaural stimulation. B. Inputs to binaural neuron. Activity of each input is synchronized to the tone at the corresponding ear with SC as indicated. C. Action potentials of the binaural neuron divided into those elicited by ipsilateral monaural coincidences, binaural coincidences, and contralateral monaural coincidences. D. Proportions of action potentials elicited in the three different ways. E. Ipsilateral, contralateral, and interaural-phase SCs for each group of action potentials. F. Equations for aggregate ipsilateral, aggregate contralateral, and aggregate interaural-phase SCs. G. The product of the aggregate SCs to tones derived from the binaural-beat stimulus is approximately equal to the interaural-phase SC.

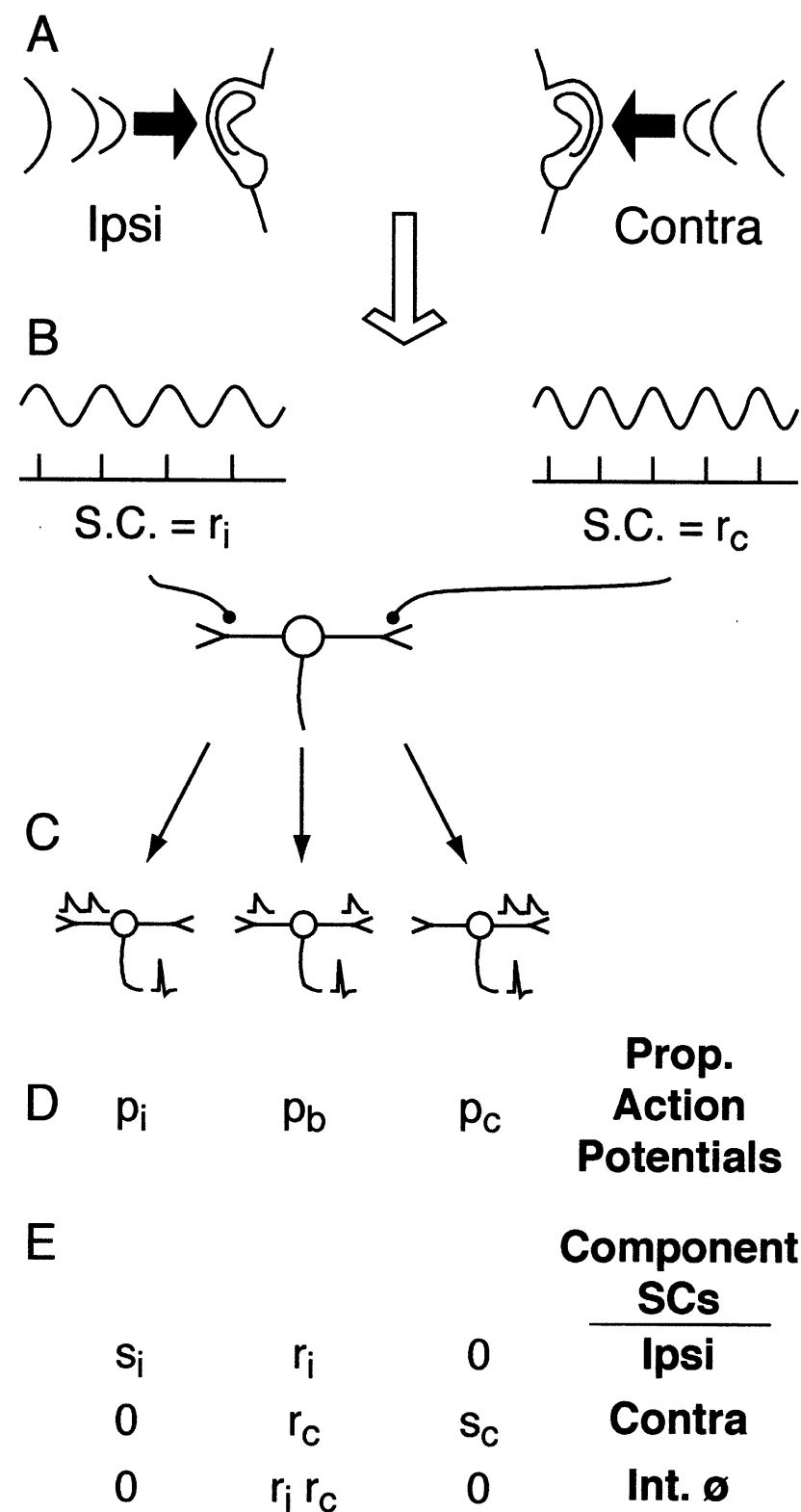

\section{F Aggregate SCs}

$$
\begin{aligned}
& r_{i b}=p_{i} s_{i}+p_{c} \times 0+p_{b} r_{i} \\
& =\left(1-p_{c}\right) r_{i}+p_{i}\left(s_{i}-r_{i}\right) \quad \text { Ipsi } \\
& \simeq\left(1-p_{c}\right) r_{i} \\
& r_{c b} \simeq\left(1-p_{i}\right) r_{c} \\
& r_{b}=p_{i} \times 0+p_{c} \times 0+p_{b} r_{i} r_{c} \\
& =\left[1-\left(p_{i}+p_{c}\right)\right] r_{i} r_{c} \\
& \text { Contra } \\
& \text { Int. } 0 \\
& G r_{i b} r_{c b} \simeq r_{b}+p_{i} p_{c} r_{i} r_{c} \simeq r_{b}
\end{aligned}
$$


and the number of monaural coincidences. When there are no monaural coincidences, the aggregate SC will be dominated by the contribution from binaural coincidences and will be about the same as the input SC. This is the case in the computer modeling of the Yin and Chan (1990) neuron (Fig. 6), for which $\alpha$ is zero (Table 1 ), and hence the number of monaural coincidences is zero as well (Fig. 7C). In this case, the aggregate SC $(0.83$ for ipsilateral tones, 0.81 for contralateral tones) is about the same as the input SC (0.88, not shown). Even when the parameters permit monaural coincidences to occur, the SC of the model neuron differs little from the input SC. This is because a substantial proportion of the action potentials still arise from binaural coincidences. In the modeling of the Goldberg and Brown (1969) neuron (Fig. 4), monaural coincidences occur because $\alpha$ is not zero. Nevertheless, the SC of the neuron differs little from the input SC (0.80, not shown). For this neuron, the SC to monaural ipsilateral stimulation is slightly larger than the SC to monaural contralateral stimulation even though the input $\mathrm{SC}$ is the same on both sides. This is because the spontaneous rate is lower on the unstimulated side during ipsilateral stimulation (Table 1), resulting in a larger proportion of monaural coincidences from the driven side. In any event, the analytic model indicates that the aggregate SC to monaural stimulation is typically similar to that of the input.

When a binaural-beat stimulus is applied to this model, the response is more complex (Fig. 9). The action potentials generated by the neuron can be divided into the same three groups as for monaural stimulation (Fig. 9C). The proportions of action potentials in each group are, in general, different from the proportions for monaural stimulation (Fig. 9D). Action potentials derived from monaural coincidences at either input will be synchronized to that input but not to the other input, and these action potentials will not be sensitive to interaural-phase differences (Fig. 9E, left and right columns). Action potentials that are a result of binaural coincidences will be synchronized to both ipsilateral and contralateral tones and will also be sensitive to interaural phase differences (Fig. 9E, middle column). The ipsilateral and contralateral SCs of the action potentials that are a result of binaural coincidences will be equal to the SCs of the respective inputs (Fig. 9E, middle column, first and second rows), for reasons similar to those given above for monaural stimulation. The interaural-phase SC of these action potentials will be given by the convolution principle (Fig. 9E, middle column, third row).

The aggregate ipsilateral SC $\left(r_{i b}\right)$ of the neuron will be given by a linear combination of the SCs of the three components (Fig. 9F, Ipsi, first expression).
This SC can be shown to be equal to the sum of two terms (Fig. 9F, Ipsi, second expression) by observing that the sum of the proportions of action potentials in each component must equal unity and by rearranging the original terms. The second of these terms $\left[p_{i}\left(s_{i}-r_{1}\right)\right]$ is the product of two other terms. The first of these other terms denotes the proportion of ipsilateral monaural coincidences, which is relatively small. The second is the difference between the ipsilateral SCs of action potentials arising from monaural and binaural coincidences, which is also small. Thus, the product of these two terms will be even smaller and can be discarded (Fig. 9F, Ipsi, third expression). The expression for the aggregate ipsilateral SC thus reduces to the SC of the ipsilateral input reduced by the proportion of action potentials that are a result of contralateral monaural coincidences. These action potentials are not synchronized to the ipsilateral tone and cannot contribute to the ipsilateral SC. A similar argument yields a corresponding expression for the aggregate contralateral SC $\left(r_{c b}\right)$ (Fig. 9F, Contra).

The aggregate interaural-phase $\mathrm{SC}$ is given by a linear combination of the interaural-phase $\mathrm{SC}$ of the three components (Fig. 9F, Int. $\phi$ ). It is, in effect, the ideal interaural-phase SC predicted by the convolution principle reduced by the proportion of action potentials elicited by monaural coincidences. The aggregate interaural-phase $\mathrm{SC}$ is therefore less than the product of SCs derived from responses to monaural tones because the latter are similar to the input SCs.

For this model, the product of the SCs to the tones derived from binaural-beat stimuli can be obtained by multiplying the simplified expressions for $r_{i b}$ and $r_{c b}$ (Fig. 9G). A rearrangement of terms reveals that this expression differs from the aggregate interauralphase SC by only a higher-order term. Thus, the product of the SCs to tones derived from binauralbeat stimuli yields a close approximation to the interaural-phase SC, whereas the product of the SCs derived from responses to monaural tones does not.

The simplified expressions for $r_{i b}$ and $r_{c b}$ (Fig. 9F) can be applied to the response shown in Figure 6 . The monaural SCs of this neuron imply that the total number of monaural coincidences $\left(p_{i}+p_{c}\right)$ is $\sim 12 \%$, whereas the modeling assumed it to be zero. The proportion of binaural coincidences for this response was therefore $\sim 88 \%$. When these equations are applied to the SCs of the full samples of neurons in the MSO of the cat and rabbit, the smallest proportion of binaural coincidences during a binaural-beat stimulus is $\sim 40 \%$. This suggests that the largest realistic value for the parameter $\alpha$ is $\sim 0.2$ (Fig. 7C, dashed lines). At these values of $\alpha$, the interaural-phase SC is relatively close to that predicted by the product of the mon- 
aural SCs derived from the response to the binauralbeat stimulus (Fig. 7B, arrowhead).

\section{DISCUSSION}

The computer and analytic modeling we have performed indicates that the SC of a neuron in the MSO to a tone presented monaurally more closely reflects the SC of the input fibers than the SC to the same tone presented as part of a binaural-beat stimulus. The latter is smaller because of the contribution of action potentials elicited by monaural coincidences at the opposite input that are unsynchronized to the tone under consideration. During a binaural-beat stimulus, this input is activated and the number of unsynchronized action potentials is relatively large. During monaural stimulation, the unstimulated input is activated only sporadically by spontaneous activity, and there are few unsynchronized action potentials. The modeling also indicates that the product of the SCs derived from the binaural-beat stimulus is closer to the measured interaural-phase SC than the product of the SCs derived from monaural stimulation because the interaural-phase SC is also reduced by the occurrence of monaural coincidences. The reduction in SCs derived from the binaural-beat stimulus is such that their product almost exactly matches the interaural-phase SC.

The modeling provides evidence that neurons in the MSO do not act as strict cross correlators, but they instead generate an output that is a degraded version of cross correlation. Previous physiological studies have generally supported the idea that MSO neurons act as coincidence detectors (Batra et al. 1997b; Crow et al. 1978; Goldberg and Brown 1969; Grothe and Sanes 1994; Joris 1996; Moushegian et al. 1975; Spitzer and Semple 1995; Yin and Chan 1990). These studies have amply demonstrated that the ITD eliciting maximal discharge is equal to the delay in response to stimulation of either ear. However, only two studies performed more refined tests to examine whether the MSO is a cross correlator (Batra et al. 1997b; Yin and Chan 1990). Both studies generally supported cross correlation. However, the study by Yin and Chan (1990) performed only a qualitative assessment on a few neurons and did note some deviations. The study by Batra et al. (1997b) tested a large sample of neurons using the present methodology, but it employed monaural SCs based on responses to binaural-beat stimuli. The modeling we have performed here indicates that these SCs underestimate the SCs of the inputs to the neuron. Thus, relative to the SCs of the inputs, the tuning of an MSO neuron to ITDs is broader than expected by cross correlation.
In the MSO, the widths of spatial receptive fields as derived from the ITD functions are determined by multiple factors. The central factor is how tightly the discharge of incoming axons is synchronized to the waveform at the ear. This, in turn, is governed by the directional polarization of inner hair cells that results in a rectification process in the inner ear, and by neural processing in the cochlear nucleus that increases the degree of synchrony (Joris et al. 1994). In the MSO, there are processes that can degrade synchrony to the waveform. One of these is the variability in the lengths of different axons from the cochlear nucleus innervating the same region of the MSO (Beckius et al. 1999). In the present report, we have found that a second factor is also likely to play a role, namely, the tendency of neurons in the MSO to discharge occasionally when inputs from only one side are activated.

The tendency of many neurons in the MSO to respond to monaural stimulation might be thought in and of itself to indicate the occurrence of monaural coincidences and a deviation from cross correlation. However, modeling indicates that monaural responses can also arise by coincidences between action potentials at the stimulated input and spontaneous action potentials at the unstimulated input (Colburn et al. 1990). Such a scheme does not produce deviations from cross correlation because all coincidences that occur are binaural coincidences. The present work suggests that for most neurons in the MSO, at least a part of the monaural responses arises from monaural coincidences.

In the Colburn et al. (1990) model, a substantial portion of the response to monaural stimulation is a result of binaural coincidences between the driven input and spontaneous activity on the idle input. Such a high proportion of binaural coincidences appears necessary to simultaneously fit both binaural and monaural responses of the same neuron. However, this requires relatively high levels of spontaneous activity compared to the rates on the driven input. Thus, just how monaural responses arise in the MSO remains somewhat unclear.

The data and the modeling we have performed suggest that most neurons in the MSO have a mechanism for reducing the proportion of action potentials elicited by monaural coincidences. The specific model we have considered here uses a refractory input to reduce the tendency of the model neuron to discharge in response to temporal summation of monaural inputs. Even a neuron that discharged relatively vigorously to monaural stimuli still required model inputs with relatively strong refractory periods to fit both the monaural and binaural responses. However, it is unlikely that the MSO really uses a refractory period to limit the number of monaural coincidences because most neurons probably receive 
multiple inputs from one side. Suppression of monaural coincidences could be the result of combinatorial considerations in that binaural coincidences may be more likely than monaural coincidences (Colburn et al. 1990). Suppression may also be accomplished by the known segregation of the ipsilateral and contralateral inputs onto different dendrites, coupled with shunting of the postsynaptic current (Agmon-Snir et al. 1998). Yet another possibility is that inhibition occurs at ITDs at which binaural coincidences do not, counteracting the dispersive effects of monaural coincidences. There is ample evidence that inhibitory inputs are present and functional (Adams and Mugnaini 1990; Banks and Smith 1992; Brand et al. 2002; Cant and Hyson 1992; Covey et al. 1991; Grothe and Sanes 1993, 1994; Kiss and Majorossy 1983; Kuwabara and Zook 1992; Smith 1995; Spangler et al. 1985).

The present study illustrates the pitfalls of inferring the details of the timing of inputs to a neuron from the responses of the neuron itself, as pointed out by Irvine et al. $(1995,2001)$ and mentioned in the Introduction. The close relationship between the ITD that elicits maximal discharge in a neuron of the MSO and the relative timing of its responses to monaural stimulation of each ear has been taken as evidence that changes in the timing of the inputs to any auditory neuron are reflected in changes to its own responses. If the mean phase of the response is considered to be a first-order timing effect and the SC a second-order effect, then it appears that this assumption holds well for first-order effects but not as well for the second-order effect. The present study sounds an additional note of caution in making such inferences.

For one neuron of the MSO of the cat, the SCs derived from binaural-beat stimuli consistently underestimated the interaural-phase SC (Fig. 3B, neuron associated with unfilled circle at upper left). A deviation of this kind is difficult to mimic with the model of Colburn et al. (1990). Such a deviation has been taken as evidence that the neuron in question is a higher-order neuron and not a primary generator of sensitivity to ITDs (Batra and Fitzpatrick 2002; Batra et al. 1997b). It is therefore possible that higher-order neurons are present within the MSO, as well as the neurons that are primary generators of ITD sensitivity. Alternatively, there may be processes within the MSO that are not encompassed by the simple model we have employed.

\section{ACKNOWLEDGMENTS}

We are pleased to acknowledge the efforts of Drs. S. Kuwada, D.C. Fitzpatrick, and J.C.K. Chan during the data collection and initial analysis. Ravi Kochhar helped on computer matters and Y. Zhou suggested the rationale for the convolution principle based on Fourier analysis. This work was supported by NSF grant IBN 9807872 and NIH grants DC 02840. The original studies in the cat and rabbit were supported by NIH grants DC 00116 to T.C.T. Yin and DC 01366 to S. Kuwada.

\section{APPENDIX}

Here we show that a circular distribution that is the linear combination of two other distributions with identical mean directions has an SC that is a linear combination of the SCs of the initial distributions. Let the two initial distributions be $f(\phi)$ and $g(\phi)$, and the resulting distribution be $h(\phi)$ :

$$
h(\phi)=a f(\phi)+(1-a) g(\phi)
$$

The mean directions of $f(\phi)$ and $g(\phi)$ are assumed equal. As the relationship we are trying to prove involves the SCs and not the mean directions, we can assume the mean directions are zero without any loss of generality:

$$
\int f(\phi) \sin (\phi) d \phi=\int g(\phi) \sin (\phi) d \phi=0,
$$

where the integrals here and below are taken over one cycle.

The synchronization coefficient $r_{h}$ of $h(\phi)$ is given by

$$
\begin{aligned}
r_{h}^{2}= & {\left[\int h(\phi) \sin (\phi) d \phi\right]^{2} } \\
& +\left[\int h(\phi) \cos (\phi) d \phi\right]^{2} \\
= & \left\{\int[a f(\phi)+(1-a) g(\phi)] \sin (\phi) d(\phi)\right\}^{2} \\
& +\left\{\int[a f(\phi)+(1-a) g(\phi)] \cos (\phi) d(\phi)\right\}^{2} \\
= & {\left[a \int f(\phi) \sin (\phi) d \phi+(1-a) \int g(\phi) \sin (\phi) d \phi\right]^{2} } \\
& +\left[a \int f(\phi) \cos (\phi) d \phi+(1-a) \int g(\phi)\right. \\
& \quad \cos (\phi) d \phi]^{2}
\end{aligned}
$$

From Eq. (A1), the first two integrals in the final expression above are zero, so the equation reduces to

$$
r_{h}=a \int f(\phi) \cos (\phi) d \phi+(1-a) \int g(\phi) \cos (\phi) d \phi
$$

The SC of $f(\phi)$ is given by 


$$
r_{f}^{2}=\left[\int f(\phi) \sin (\phi) d \phi\right]^{2}+\left[\int f(\phi) \cos (\phi) d \phi\right]^{2}
$$

but using Eq. (A1) this simplifies to

$$
r_{f}=\int f(\phi) \cos (\phi) d \phi
$$

A similar expression holds for the SC for $g(\phi), r_{g}$. Substituting in Eq. (A2) yields

$$
r_{h}=a r_{f}+(1-a) r_{g}
$$

Although we have shown this result for combining two distributions, it is straightforward to extend it to three or more.

\section{REFERENCES}

Adams JC, Mugnaini E. Immunocytochemical evidence for inhibitory and disinhibitory circuits in the superior olive. Hear. Res. 49:281-298, 1990.

Agmon-Snir H, Carr CE, Rinzel J. The role of dendrites in auditory coincidence detection. Nature 393:268-208, 1998.

Banks MI, Sмітн PH. Intracellular recordings from neurobiotinlabeled cells in brain slices of the rat medial nucleus of the trapezoid body. J. Neurosci. 12:2819-2837, 1992.

Batra R, FitzPatrick DC. Processing of interaural temporal disparities in the medial division of the ventral nucleus of the lateral lemniscus. J. Neurophysiol. 88:666-675, 2002.

Batra R, Kuwada S, Fitzpatrick DC. Sensitivity to interaural temporal disparities of low- and high-frequency neurons in the superior olivary complex: I. Heterogeneity of responses. J. Neurophysiol. 78:1222-1236, 1997a.

Batra R, Kuwada S, Fitzpatrick DC. Sensitivity to interaural temporal disparities of low- and high-frequency neurons in the superior olivary complex: II. Coincidence detection. J. Neurophysiol. 78:1237-1247, 1997b.

Beckius GE, Batra R, Oliver DL. Axons from anteroventral cochlear nucleus that terminate in medial superior olive of cat: observations related to delay lines. J. Neurosci. 19:3146-3161, 1999.

Brand A, Behrend O, Marquardt T, McAlpine D, Grothe B. Precise inhibition is essential for microsecond interaural time difference coding. Nature 417:543-547, 2002.

Cant NB, Hyson RL. Projections from the lateral nucleus of the trapezoid body to the medial superior olivary nucleus in the gerbil. Hear. Res. 58:26-34, 1992.

Carr CE, Konishi M. A circuit for detection of interaural time differences in the brain stem of the barn owl. J. Neurosci. 10:32273246, 1990.

Colburn HS, Han Y-A, Culotta CP. Coincidence model of MSO responses. Hear. Res. 49:335-346, 1990.

Covey E, Vater M, Casseday JH. Binaural properties of single units in the superior olivary complex of the mustached bat. J. Neurophysiol. 66:1080-1094, 1991.

Crow G, Rupert AL, Moushegian G. Phase locking in monaural and binaural medullary neurons: implications for binaural phenomena. J. Acoust. Soc. Am. 64:493-501, 1978.

Goldberg JM, Brown PB. Response of binaural neurons of dog superior olivary complex to dichotic tonal stimuli: some physi- ological mechanisms of sound localization. J. Neurophysiol. 32:613-636, 1969.

Grothe B, SANes DH. Bilateral inhibition by glycinergic afferents in the medial superior olive. J. Neurophysiol. 69:1192-1196, 1993.

Grothe B, Sanes DH. Synaptic inhibition influences the temporal coding properites of medial superior olivary neurons: an in vitro study. J. Neurosci. 14:1701-1709, 1994.

Han Y, ColbuRn HS. Point-neuron model for binaural interaction in MSO. Hear. Res. 68:115-130, 1993.

Irvine DRF, Park VN, Mattingley JB. Responses of neurons in the inferior colliculus of the rat to interaural time and intensity differences in transient stimuli: Implications for the latency hypothesis. Hear. Res. 85:127-141, 1995.

Irvine DRF, PARK VN, MCCormick L. Mechanisms underlying the sensitivity of neurons in the lateral superior olive to interaural intensity differences. J. Neurophysiol. 86:2647-2666, 2001.

Jefreess LA. A place theory of sound localization. J. Comp. Physiol. Psychol. 41:35-39, 1948.

JorIs PX. Envelope coding in the lateral superior olive. II. Characteristic delays and comparison with responses in the medial superior olive. J. Neurophysiol. 76:2137-2156, 1996.

Joris PX, CARney LH, Smith PH, YIN TCT. Enhancement of neural synchronization in the anteroventral cochlear nucleus. I. Responses to tones at the characteristic frequency. J. Neurophysiol. 71:1022-1036, 1994.

Kiss A, Majorossy K. Neuron morphology and synaptic architecture in the medial superior olivary nucleus. Exp. Brain Res. 52:315327, 1983.

Kuwabara N, Zook JM. Projections to the medial superior olive from the medial and lateral nuclei of the trapezoid body in rodents and bats. J. Comp. Neurol. 324:522-538, 1992.

KuWAdA S, YIN TCT, WiCKESBERg RE. Response of cat inferior colliculus neurons to binaural beat stimuli: possible mechanisms for sound localization. Science 206:586-588, 1979.

Kuwada S, Batra R, FitzPatrick DC. Neural processing of binaural temporal cues. In: GILKE RH, ANDERson TR (eds) Binaural and Spatial Hearing in Real and Virtual Environments. Lawrence Erlbaum Associates, Mahwah, NJ, pp 399-425, 1997.

Mardia KV. Statistics of Directional Data Academic Press, New York, 1972.

McAlpine D, Jiang D, Palmer AR. A neural code for low-frequency sound localization in mammals. Nat. Neurosci. 4:396401, 2001.

Moushegian G, Rupert AL, Gidda JS. Functional characteristics of superior olivary neurons to binaural stimuli. J. Neurophysiol. 38:1037-1048, 1975.

Rose JE, Greenwood DD, Goldberg JM, Hind JE. Some discharge characteristics of single neurons in the inferior colliculus of the cat. I. Tonotopical organization, relation of spike-counts to tone intensity, and firing patterns of single elements. J. Neurophysiol. 26:294-320, 1963.

Sмгтн PH. Structural and functional differences distinguish principal from nonprincipal cells in the guinea pig MSO slice. J. Neurophysiol. 73:1653-1667, 1995.

Smith PH, JorIs PX, YIN TCT. Projections of physiologically characterized spherical bushy cell axons from the cochlear nucleus of the cat: evidence for delay lines to the medial superior olive. J. Comp. Neurol. 331:245-260, 1993.

Spangler KM, Warr WB, Henkel CK. The projections of principal cells of the medial nucleus of the trapezoid body in the cat. J. Comp. Neurol. 238:249-262, 1985.

Spitzer MW, Semple MN. Neurons sensitive to interaural phase disparity in gerbil superior olive: diverse monaural and temporal response properties. J. Neurophysiol. 73:1668-1690, 1995 . 
SPITZER MW, SEMPLE MN. Transformation of binaural response properties in the ascending auditory pathway: influence of time-varying interaural phase disparity. J. Neurophysiol. 80:3062-3076, 1998.

Stern RM, Trahiotis C. Models of binaural perception. In: GiLKE RH, Anderson TR (eds) Binaural and Spatial Hearing in Real and Virtual Environments Lawrence. Erlbaum Associates, Mahwah, pp 499-531, 1997.

YIN TCT. Neural mechanisms of encoding binaural localization cues in the auditory brainstem. In: Oertel D, FAY RR, Popper AN (eds) Springer Handbook of Auditory Research-Integrative
Functions in the Mammalian Auditory Pathway. Springer-Verlag, New York, pp 99-159, 2002.

YIN TCT, Chan JCK. Interaural time sensitivity in medial superior olive of cat. J. Neurophysiol. 64:465-488, 1990.

YIN TCT, KUwADA S. Binaural interaction in low-frequency neurons in inferior colliculus of the cat. III. Effects of changing frequency. J. Neurophysiol. 50:1020-1042, 1983.

Young SR, Rubel EW. Frequency-specific projections of individual neurons in chick brainstem auditory nuclei. J. Neurosci. 3:1373$1378,1983$. 